\title{
Macrophage-Inducible C-Type Lectin Signaling Exacerbates Acetaminophen-Induced Liver Injury by Promoting Kupffer Cell Activation in Mice ${ }^{\mathrm{s}}$
}

\author{
Jing Zhao, Jong-Won Kim, Zixiong Zhou, Jing Qi, Weishun Tian, Chae Woong Lim, \\ Kang Min Han, and Bumseok Kim
}

Biosafety Research Institute and College of Veterinary Medicine, Jeonbuk National University, Iksan, Jeonbuk, Republic of Korea (J.Z., J.-W.K., Z.Z., J.Q., W.T., C.W.L., B.K.); Department of Pathology, Dongguk University Ilsan Hospital, Goyang, Republic of Korea (K.M.H.); and College of Animal Science and Technology, Henan University of Science and Technology, Luoyang, Henan, People's Republic of China (J.Z.)

Received April 23, 2020; accepted November 18, 2020

\section{ABSTRACT}

Overdose of acetaminophen (APAP) has become one of the most frequent causes of acute liver failure. Macrophageinducible C-type lectin (Mincle) acts as a key moderator in immune responses by recognizing spliceosome-associated protein 130 (SAP130), which is an endogenous ligand released by necrotic cells. This study aims to explore the function of Mincle in APAP-induced hepatotoxicity. Wild-type (WT) and Mincle knockout (KO) mice were used to induce acute liver injury by injection of APAP. The hepatic expressions of Mincle, SAP130, and Mincle signaling intermediate (Syk) were markedly upregulated after the APAP challenge. Mincle KO mice showed attenuated injury in the liver, as shown by reduced pathologic lesions, decreased alanine aminotransferase and aspartate aminotransferase levels, downregulated levels of inflammatory cytokines, and decreased neutrophil infiltration. Consistently, inhibition of Syk signaling by GS9973 alleviated APAP hepatotoxicity. Most importantly, Kupffer cells (KCs) were found as the major cellular source of Mincle. The depletion of KCs abolished the detrimental role of Mincle, and the adoptive transfer of WT KC to Mincle KO mice partially reversed the hyporesponsiveness to hepatotoxicity induced by APAP. Furthermore, the expression levels of interleukin (IL) $-1 \beta$ and neutrophil-attractant CXC chemokines were substantially lower in KCs isolated from APAP-treated Mincle KO mice compared with those from WT mice. Similar results were found in primary Mincle KO KCs treated with a ligand of Mincle (trehalose-6,6-dibehenate) or in conditioned media obtained from APAP-treated hepatocytes. Collectively, Mincle can regulate the inflammatory response of $\mathrm{KCs}$, which is necessary for the complete progression of hepatotoxicity induced by APAP.

\section{SIGNIFICANCE STATEMENT}

Acetaminophen (APAP) overdose is becoming a main cause of drug-induced acute liver damage in the developed world. This study showed that macrophage-inducible C-type lectin (Mincle) deletion or inhibition of Mincle downstream signaling attenuates APAP hepatotoxicity. Furthermore, Mincle as a modulator of Kupffer cell activation contributes to the full process of hepatotoxicity induced by APAP. This mechanism will offer valuable insights to overcome the limitation of APAP hepatotoxicity treatment.
This research was supported by Basic Science Research Program through the National Research Foundation (NRF) funded by the Ministry of Education [2017R1D1A3B03030521] and by the Korean government (MSIT, Ministry of Science and ICT) [2020R1A2C1007178]. J.Z. also gratefully acknowledges the financial support from the China Scholarship Council (CSC) [File No. 201608260078]

https://doi.org/10.1124/molpharm.120.000043.

S This article has supplemental material available at molpharm. aspetjournals.org.

\section{Introduction}

As a widely used analgesic and antipyretic drug, acetaminophen (APAP) has an excellent safety profile at therapeutic doses. However, acute or accumulated excessive APAP can evoke severe liver damage, resulting in acute liver failure (Jaeschke and Bajt, 2006; Chun et al., 2009). Epidemiologic research showed that half of the patients with APAP overdoses were cases of unintentional overdosing when more than one APAP-containing analgesic was taken (Larson et al.,

ABBREVIATIONS: ALT, alanine aminotransferase; APAP, acetaminophen; AST, aspartate aminotransferase; Ct, cycle threshold; CXCL, CXC motif chemokine ligand; DAMP, danger-associated molecular pattern; DC, dendritic cell; GAPDH, glyceraldehyde-3-phosphate dehydrogenase; GdCI3, gadolinium chloride hexahydrate; GSH, glutathione; IL, interleukin; KC, Kupffer cell; KO, knockout; LDH, lactate dehydrogenase; Ly6G, lymphocyte antigen 6 complex locus G6D; Mincle, macrophage-inducible C-type lectin; MPO, myeloperoxidase; NAPQI, N-acetyl-p-benzoquinone imine; NLRP3, nucleotide-binding domain and leucine-rich repeat protein 3; NPC, nonparenchymal cell; PCR, polymerase chain reaction; pSyk, phophorylated spleen tyrosine kinase; qPCR, quantitative real-time polymerase chain reaction; SAP130, spliceosome-associated protein 130; Syk, spleen tyrosine kinase; TDB, trehalose-6,6-dibehenate; TNF- $\alpha$, tumor necrosis factor- $\alpha$; TUNEL, terminal deoxynucleotidyl transferase-mediated dUTP nick-end labeling; WT, wild-type. 
2005). Recently, programmed necrosis has been better termed as a cell death mode induced by APAP (Jaeschke et al., 2019). APAP hepatotoxicity is triggered by $N$-acetyl- $p$-benzoquinone imine (NAPQI), which is a reactive metabolite of the cytochrome P450 isoenzyme, especially CYP2E1 (Zaher et al., 1998; Chen et al., 2020). The toxicity of NAPQI is relieved by binding to hepatic glutathione (GSH). Once GSH is exhausted, excessive NAPQI eventually leads to mitochondrial oxidative stress and hepatocellular necrosis through binding to mitochondrial proteins (Larsen and Wendon, 2014). Based on the insights into this mechanism, the drug $N$-acetylcysteine, which is used in the synthesis of GSH, has been introduced to treat the patients suffering from APAP hepatotoxicity (Prescott et al., 1977). In particular, the benefit of $N$-acetylcysteine is greater when it is administered as soon as possible after APAP overdose (Smilkstein et al., 1988; Lancaster et al., 2015).

Hepatocellular necrosis causes the secretion of various danger-associated molecular patterns (DAMPs), including histones, high-mobility group box 1 , and DNA. DAMPs are host biomolecules that can be recognized by nonparenchymal cells (NPCs), especially resident hepatic Kupffer cells (KCs), resulting in the activation of NPCs in an autocrine manner (Martin-Murphy et al., 2010). The formation of the inflammasome by $\mathrm{KCs}$ then activates the innate immune system, causing substantial infiltration of neutrophils and macrophages to the inflammation site (Lawson et al., 2000; Holt et al., 2008). The systemic inflammation along with consequently advancing hepatocellular necrosis causes acute liver failure, leading to mortality.

As one of the C-type lectin superfamily members, macrophageinducible C-type lectin (Mincle) is found to be detected on myeloid cells such as macrophages, neutrophils, and dendritic cells (DCs). Mincle signaling is necessary for the formation of the innate immune system, and in particular, it was found to be strongly induced when stimulated by inflammatory materials (Matsumoto et al., 1999; Richardson and Williams, 2014). As a transmembrane receptor, Mincle has an extracellular carbohydrate recognition domain, which mediates interaction with immunoreceptor tyrosine-based activation motif (ITAM)-bearing adaptor molecule Fc receptor $\gamma$-chain (Yamasaki et al., 2008). Furthermore, Mincle is activated by the mycobacterial cell wall component trehalose-6,6-dimycolate and the synthetic analog trehalose6,6-dibehenate (TDB) (Ishikawa et al., 2009; Lin et al., 2017). Additionally, activation of Mincle can be induced by the release of a small nuclear ribonucleoprotein component called spliceosome-associated protein 130 (SAP130) (Brown, 2008; Yamasaki et al., 2008). Mincle signaling induces inflammatory responses through the recruited caspase recruitment domain family member 9 (CARD9) adaptor protein and phosphorylated Syk, leading to inflammatory responses with the generation of cytokines including TNF- $\alpha$ and IL-6 (Brown, 2008).

Recently, a large amount of evidence has indicated that the Mincle signaling pathway plays an important role in noninfectious inflammatory disorders (de Rivero Vaccari et al., 2015; He et al., 2015). Moreover, studies have revealed that Mincle deletion protects against ethanol or concanavalin A-induced liver injury (Greco et al., 2016; Zhou et al., 2016; Kim et al., 2018). Currently, the effects of Mincle signaling on APAP hepatotoxicity have not been completely explored yet.
Therefore, we investigated the role of Mincle in the progression of APAP hepatotoxicity and investigated the underlying mechanism.

\section{Materials and Methods}

Experimental Mice and Protocol. Wild-type (WT) male C57BL/ $6 \mathrm{~N}$ mice ( 8 weeks) were obtained from Taconic Farms, Inc. (Samtako Bio Korea, O-San, Republic of Korea). Mincle knockout (KO) mice were kindly provided by Prof. Young-Joon Kim (Yonsei University, Republic of Korea) and backcrossed on C57BL/6N mice for at least 10 generations (Lee et al., 2012). Mice were kept in a standard condition (24 $\pm 2{ }^{\circ} \mathrm{C}, 50 \% \pm 5 \%$ humidity, 12 -hour light/dark cycle). Animal management procedures and breeding facilities have been approved by the requirements of the Animal Care and Ethics Committees of Jeonbuk National University.

To induce liver injury, animals were fasted for 16 hours and intraperitoneally injected with the indicated dose of APAP (150$500 \mathrm{mg} / \mathrm{kg}$ ) (Sigma-Aldrich, St Louis, MO). In the selected experiments, mice were pretreated with an oral administration of a Syk inhibitor, entospletinib (GS9973; Cayman Chemical, Ann Arbor, MI) (Currie et al., 2014), or vehicle control 1 hour before APAP injection. To reveal the role of macrophages in APAP-induced liver injury, gadolinium chloride hexahydrate $\left(\mathrm{GdCl}_{3}\right.$; Sigma-Aldrich) was administered intravenously to mice $(10 \mathrm{mg} / \mathrm{kg})$ to inhibit or deplete the $\mathrm{KCs}$ (Chatterjee et al., 2013; Merlin et al., 2016). Subsequently, mice were anesthetized after 24 hours of APAP injection, and blood was collected by cardiac puncture. Sizes of the tested animal groups in different experiments were chosen according to standard guidelines (Michel et al., 2020) and dictated by the available number of mice of suitable strains, age, and weight. Livers were harvested for gene expression and histologic analysis.

Hepatocyte and KC Isolation. Primary hepatocytes from WT or Mincle KO mice were prepared by collagen perfusion. Briefly, collagenase 1 passing through the portal vein was used to digest mouse livers. The digested cells were suspended and centrifuged at $50 \mathrm{~g}$ for 3 minutes. The pellet representing hepatocytes was collected and resuspended after centrifugation, filtered, and washed several times. Cellular viability and cell number were measured by the trypan blue dye exclusion method (Sigma-Aldrich).

Primary KCs were isolated from mouse liver (Zhao et al., 2020). Briefly, the digested liver cells were centrifuged at $50 \mathrm{~g}$ for 3 minutes, and NPCs were collected from the supernatant. Then, Percoll density gradient centrifugation was performed for the enrichment of KCs. KC fractions were collected from the interface of $40 \%$ and $70 \%$ Percoll after centrifugation at $750 \mathrm{~g}$ for 20 minutes without brake. The positive selection of KCs was performed by magnetic cell sorting using the anti-F4/80 antibody (catalog number 13-4801-85; e-Biosciences).

Adoptive Transfer of KCs. Macrophage adoptive transfer was performed as described previously (Wu et al., 2012; Chatterjee et al., 2013; Greco et al., 2016). Briefly, isolated F4/80 $/ 0^{+} \mathrm{KCs}$ from WT mice were injected intravenously into macrophage-depleted WT or Mincle KO recipients $\left(1 \times 10^{6}\right.$ cells/mouse in $200 \mu$ l sterile PBS per injection). After 24 hours, mice were injected with $300 \mathrm{mg} / \mathrm{kg}$ APAP.

Histopathologic and Immunohistochemical Examination. Mouse hepatic tissue was routinely fixed, processed, and embedded in paraffin wax. Tissue sections $(5 \mu \mathrm{m})$ were prepared. For histologic analysis, H\&E staining or terminal deoxynucleotidyl transferase-mediated dUTP nick-end labeling (TUNEL) staining was performed on tissue sections, and histologic observation was done by light microscopy (BX51; Olympus Corp., Tokyo, Japan). Positive areas of TUNEL-labeled cells were determined on a computerized grid as described previously (Zhao et al., 2020). For immunohistochemical staining, tissue sections were stained with anti-mouse myeloperoxidase (MPO, catalog number ab9535; Abcam), which is a neutrophil marker, as described previously (Kim et al., 2018). Slide images were taken under a light 
TABLE 1

Sequences of mouse primers used in qPCR

\begin{tabular}{|c|c|c|}
\hline Gene & Primer Sequences, Forward & Primer Sequences, Reverse \\
\hline Mincle & 5'-AACCCACTCTATCTGCTCAGTGCTT-3' & 5'-CCAGCATGAATGGCATGGA-3' \\
\hline IL- $1 \beta$ & 5'-CTCGCAGCAGCACATCAACA-3' & 5'-CCACGGGAAAGACACAGGTA-3' \\
\hline CYP2E1 & 5'-AAGCGCTTCGGGCCAG-3' & 5'-TAGCCATGCAGGACCACGA-3' \\
\hline CXCL1 & 5'-TGCACCCAAACCGAAGTC-3' & 5'-GTCAGAAGCCAGCGTTCACC-3' \\
\hline CXCL2 & 5'-GCCAAGGGTTGACTTCAAGAACA-3' & 5'-AGGCTCCTCCTTTCCAGGTCA-3' \\
\hline GAPDH & 5'-ACGGCAAATTCAACGGCACAG-3' & 5'-AGACTCCACGACATACTCAGCAC-3' \\
\hline
\end{tabular}

microscope (BX-51; Olympus Corp.) in a blinded manner and subjected to digital image analysis (analySIS TS; Olympus Corp.).

Biochemical Measurements. Commercial kits were used to measure the serum levels of alanine aminotransferase (ALT) and aspartate aminotransferase (AST) (ASAN Pharmaceutical, Hwasung, Republic of Korea). Samples of serum were prepared by centrifugation $\left(1000 g, 15\right.$ minutes, $4^{\circ} \mathrm{C}$ ). Hepatic GSH levels were measured by a GSH quantification kit (Dojindo, Kumamoto, Japan). An EMax spectrophotometer (Molecular Devices, Sunnyvale, CA) was used to determine the optical density at a wavelength of 490 or $412 \mathrm{~nm}$.

Enzyme-Linked Immunosorbent Assay. Protein extracted from the liver was used for quantification of the levels of cytokines. After centrifugation at $13,000 \mathrm{~g}$ for 15 minutes at $4^{\circ} \mathrm{C}$, the precipitate was discarded, and protein concentration in the supernatant was determined using the Pierce Bicinchoninic acid (BCA) protein assay kit (Thermo Fisher Scientific, Inc.). The levels of each respective cytokine were determined using the commercial ELISA kits
(eBioscience, San Diego, CA). All experiments were performed according to the manufacturer's instructions.

Flow Cytometric Analysis. NPCs isolated from the liver were stained with Fc blocking reagent (catalog number 553142; BD Biosciences, Franklin Lakes, NJ) for 20 minutes, followed by incubation with fluorescently conjugated anti-CD11b antibody (cat. no. 550993; BD Biosciences) and anti-Ly6G antibody (cat. no. 12-9668-82; e-Biosciences) on ice for 30 minutes. Each sample in experiments was performed by an Accuri C6 flow cytometer (BD Biosciences) and analyzed by BD CFlowPlus software version 1.0.227.4 (BD Biosciences). Target cell frequencies were expressed as the percentage for a specific cell subset.

Lactate Dehydrogenase Assay. Lactate dehydrogenase (LDH) assay was used to evaluate the hepatocellular cytotoxicity based on the measurement of the levels of $\mathrm{LDH}$ released into the culture medium with Cytotoxicity Detection Kit (Sigma-Aldrich). An EMax spectrophotometer (Molecular Devices) was used to determine the optical density at a wavelength of $490 \mathrm{~nm}$.
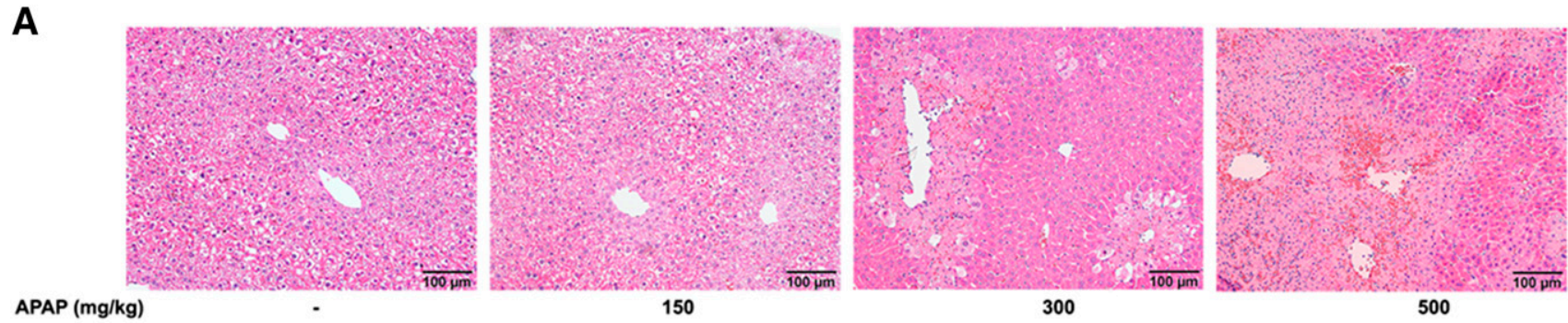

B
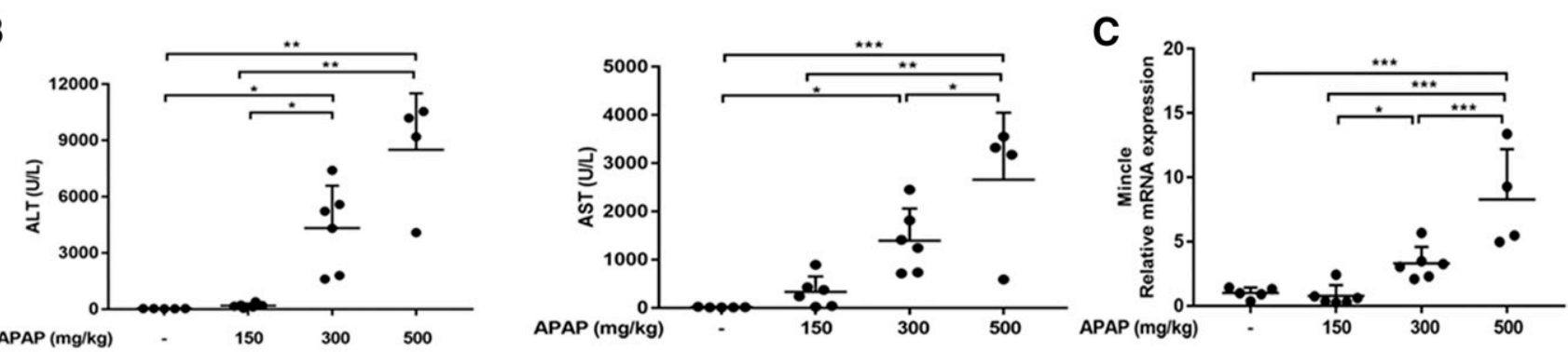

\section{D}
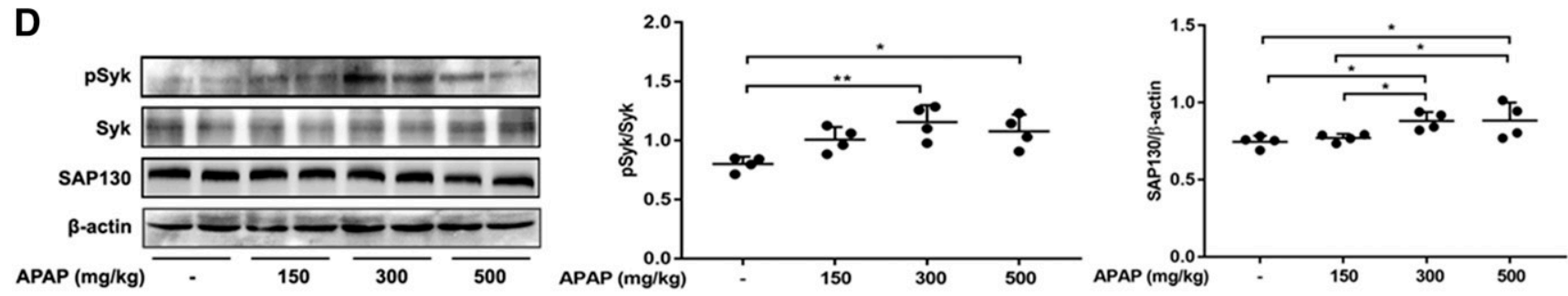

Fig. 1. The expression of Mincle signaling genes in APAP-induced liver injury. WT male mice were injected with pyrogen-free PBS $(n=5)$ or APAP (150, 300 , or $500 \mathrm{mg} / \mathrm{kg})(n=6)$, and samples were harvested at 24 hours. (A) Representative histologic liver sections stained with H\&E are shown. Scale bar, $100 \mu \mathrm{m}$. (B) Serum levels of ALT and AST were measured. (C) Hepatic mRNA expression levels of Mincle were measured by quantitative real-time PCR, and data are shown as fold change compared with the relative expression of Mincle in PBS-injected mice. (D) Western blot analysis was performed using pSyk, Syk, and SAP130 antibodies. Data are presented as means \pm S.D. A one-way ANOVA with Tukey's post hoc test was performed in $(B-D)$. * $P<0.05$; $* * P<0.01 ; * * * P<0.001$ vs. the indicated groups. 
Quantitative Real-Time Polymerase Chain Reaction. The Easy-Spin Total RNA extraction kit (GeneAll, Republic of Korea) was used to extract total RNA from liver tissue or cells. cDNA was prepared by treatment with genomic DNA (gDNA) remover, followed by random primer and MultiScribe MuLV Reverse Transcriptase (Toyobo Co.). qPCR was performed on a CFX96 Real-Time PCR Detection System (Bio-Rad Laboratories, Hercules, CA) with cDNA. Glyceraldehyde-3-phosphate dehydrogenase (GAPDH) was used as an internal control to analyze the quantification. All PCR primers were purchased from Bioneer (Daejeon, Republic of Korea). PCR primer sequences are shown in Table 1. All experiments were performed according to the manufacturer's instructions.

Immunoblot Analysis. Protein from liver tissues was lysed, and the concentration was measured. After subjecting the protein to SDSPAGE and transferring to polyvinylidene difluoride membrane, blocking was prepared with $5 \%$ bovine serum albumin in Tris-buffered saline containing $0.05 \%$ Tween-20 for 1 hour at room temperature. Subsequently, the following antibodies were used at a 1:1000 dilution in a blocking buffer at $4^{\circ} \mathrm{C}$ overnight: anti-pSyk antibody (cat. no. 2710; Cell Signaling Technology), anti-Syk antibody (cat. no. 2712; Cell Signaling Technology), anti-SAP130 antibody (cat. no. GTX122554; GeneTex Inc.), anti-Mincle antibody (cat. no. D266-3; MBL International Corporation), anti-CYP2E1 antibody (cat. no. 1252; Millipore), and anti- $\beta$-actin (catalog number 3700; Cell Signaling Technology). To detect antigen-antibody complexes, peroxidase-conjugated antirabbit (cat. no. ADI-SAB-300-J; Enzo Life Sciences), anti-mouse (cat. no. ADI-SAB-100-J; Enzo Life Sciences), or anti-rat (cat. no. sc-2032; Santa Cruz Biotechnology, Inc.) secondary antibodies were prepared at a 1:3000 dilution in a blocking buffer and incubated at room temperature for 1 hour. Protein bands were visualized with an enhanced chemiluminescence detection system (Clarity Western enhanced chemiluminescence substrate; Bio-Rad) using ImageQuant LAS 500 (GE Healthcare Life Science). Expression levels of protein were quantified using the ImageQuant TL software version 8.1.

Statistical Analysis. All quantitative data obtained in experiments are subjected to statistical analyses and expressed as a mean \pm S.D. Statistical analysis was performed using two-sided unpaired Student's $t$ tests between two groups or using one-way ANOVA with
Tukey's post hoc test between three or more groups, as performed by GraphPad Prism 7.0 software (Graph Pad Software, San Diego, CA). Statistical significance was set at a value of $P<0.05$. The sample size was predetermined based on the level of variation observed in the previous experiment. The experiments were done in an exploratory manner without testing a prespecified statistical null hypothesis, and the $P$ value is explained for descriptive purposes.

\section{Results}

APAP Treatment Induces Mincle, SAP130, and pSyk Expression in Mouse Liver. Intraperitoneal injection with APAP can induce liver damage dose-dependently. It was shown that hepatocellular necrosis and serum transaminase levels increased with an increase in the dose of APAP injection (Fig. 1, A and B). To explore the effects of Mincle signaling on APAP hepatotoxicity, the expressions of Mincle, SAP130 (a ligand of Mincle), and related downstream Mincle signaling molecules (pSyk and Syk) were measured. The mRNA expression of Mincle was markedly increased in the liver when mice were treated with either 300 or $500 \mathrm{mg} / \mathrm{kg}$ APAP (Fig. 1C). Additionally, protein levels of SAP130 and pSyk were substantially elevated, and mice treated with a dose of APAP at $300 \mathrm{mg} / \mathrm{kg}$ showed the highest hepatic protein levels of SAP130 and Syk phosphorylation (Fig. 1D). The Ct value of GAPDH and band intensity of $\beta$-actin were represented (Supplemental Fig. 1). These results showed that Mincle signaling was related to APAP-induced liver injury. Of note, since $500 \mathrm{mg} / \mathrm{kg}$ APAP is a lethal dose for mice and two of six mice in this group died in this experiment, a dose of APAP at $300 \mathrm{mg} / \mathrm{kg}$ was used in later experiments.

Mincle Deletion Ameliorates APAP-Induced Liver Injury. In the present study, Mincle KO mice were used to determine the role of Mincle signaling in APAP hepatotoxicity, and Western blot analysis and genotype analysis were used to
A $\quad$ wT

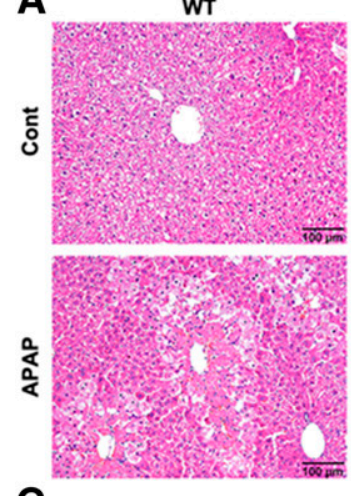

мко

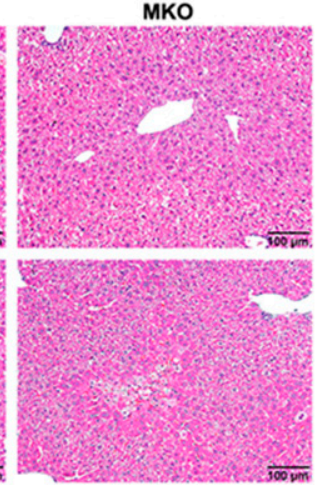

B
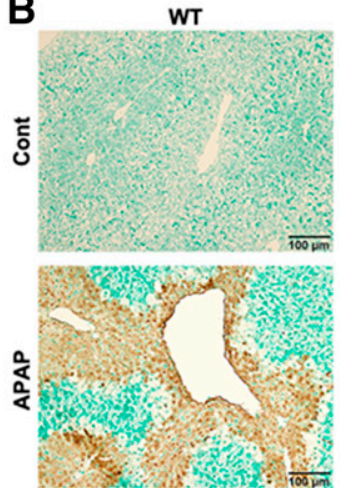

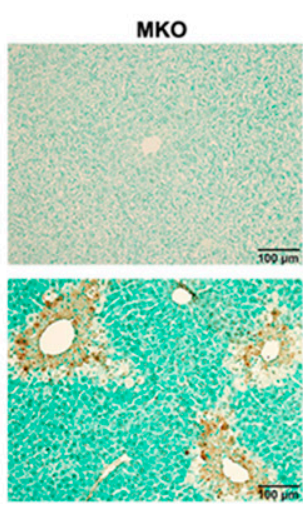

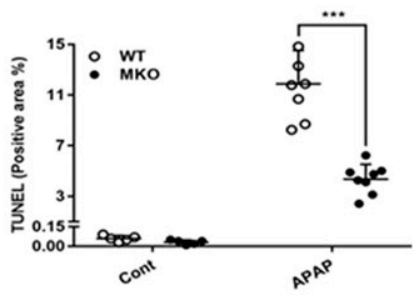
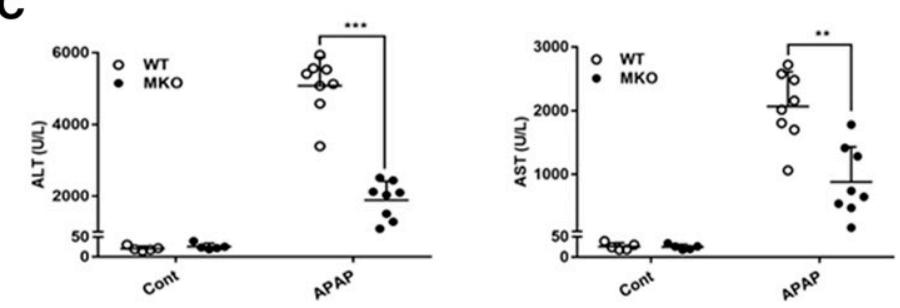

Fig. 2. The effect of Mincle deficiency in APAP-induced liver injury. Mice were administered with PBS (Cont, control $)(n=5)$ or APAP $(300 \mathrm{mg} / \mathrm{kg})(n=8)$, and samples were harvested at 24 hours. (A) Representative histologic liver sections stained with H\&E are shown. (B) Apoptosis was determined by TUNEL staining and quantification of TUNEL-positive area. (C) Serum levels of ALT and AST were measured for each cohort. Data are presented as means \pm S.D. A $t$ test was performed in (B and C). ${ }^{* * P}<0.01$; ${ }^{* * *} P<0.001$ vs. the indicated groups. Scale bar, $100 \mu \mathrm{m}$. MKO, Mincle KO. 


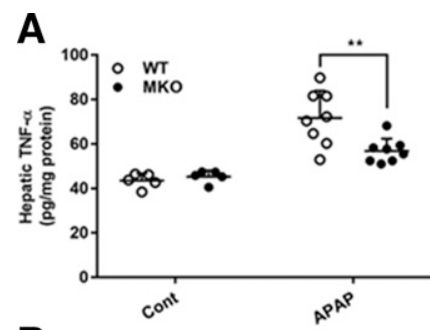

B

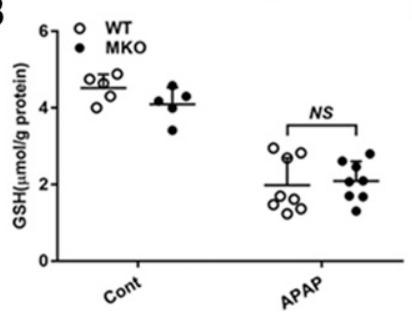

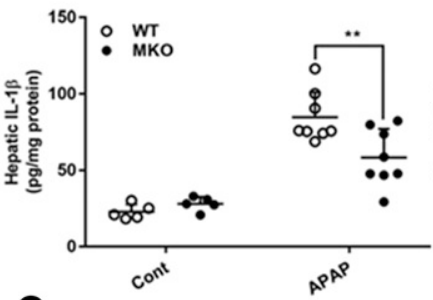

C

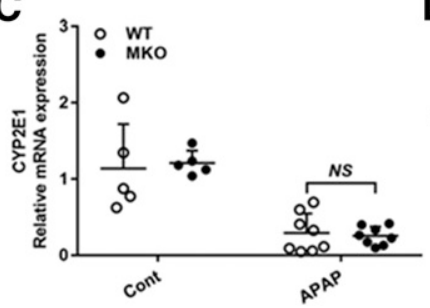

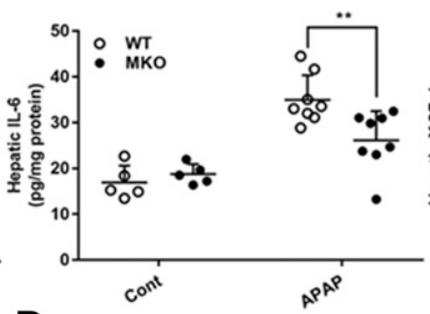

D

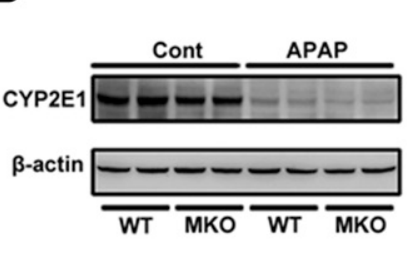

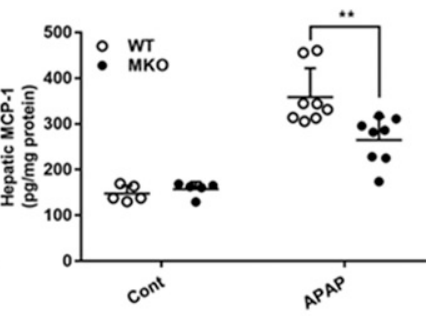

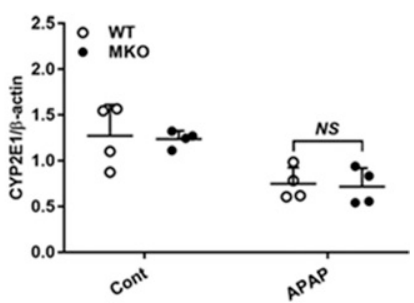

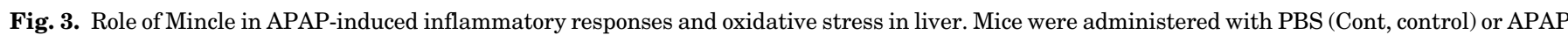

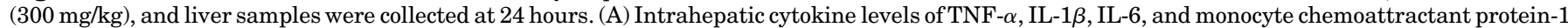

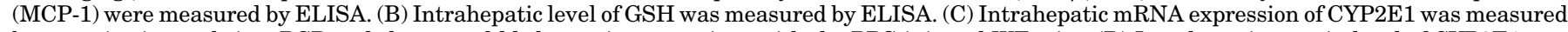

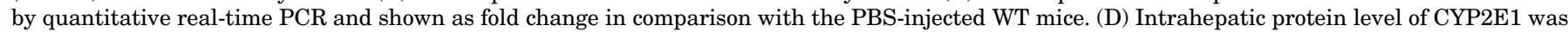

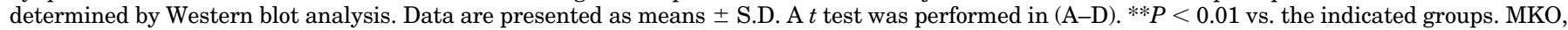
Mincle KO; NS, no significant difference.

determine the generation of Mincle KO mice (Supplemental Fig. 2; Supplemental Table 1). After injecting APAP into WT and Mincle KO mice, the results showed that hepatic necrosis in Mincle KO mice was markedly decreased when compared with WT mice (Fig. 2A). Similarly, decreased TUNEL-positive nuclear bodies (Fig. 2B) and lower levels of serum aminotransferases (Fig. 2C) were found in APAP-treated Mincle KO mice. These data indicated that Mincle deletion ameliorated APAP hepatotoxicity.

Mincle Increases the Release of Proinflammatory Mediators Without Altering APAP Metabolism in the Injured Liver. Liver injury induced by APAP is correlated with increased hepatic levels of proinflammatory cytokines. To examine whether mitigated toxicity in Mincle KO mice is associated with levels of proinflammatory mediators, hepatic levels of TNF- $\alpha$, IL- $1 \beta$, IL- 6 , and monocyte chemoattractant protein-1 (MCP-1) were measured by ELISA. Compared with WT mice, hepatic levels of the cytokines were decreased in Mincle KO mice (Fig. 3A). CYP2E1 is essential for APAP metabolism with a potential to convert APAP into bioactivated NAPQI, which causes depletion of antioxidants such as GSH. The hepatic level of GSH and expression of CYP2E1 were examined in WT and Mincle KO mice to investigate whether the Mincle signaling was related to APAP metabolism. However, no statistically significant difference between the two types of mice in the hepatic level of GSH and the mRNA level of CYP2E1 was observed with APAP treatment (Fig. 3, B and $\mathrm{C}$ ). Moreover, no statistically significant difference between WT and Mincle KO mice was shown in the hepatic protein levels of CYP2E1 upon APAP treatment (Fig. 3D). The $\mathrm{Ct}$ value of GAPDH and band intensity of $\beta$-actin were represented (Supplemental Fig. 3). These results suggested that Mincle affected the expression of proinflammatory mediators but did not change APAP metabolism in the pathogenesis of APAP hepatotoxicity.

Mincle Deficiency Decreases the Infiltration of Neutrophils in the Liver after APAP Treatment. It is well known that the recruitment of neutrophils to the injured site is a characteristic event in APAP hepatotoxicity. Therefore, neutrophil infiltration was evaluated in this study. The results showed that WT mice exhibited higher infiltration of intrahepatic neutrophils compared with Mincle KO mice after treatment with APAP (Fig. 4A). Consistently, immunohistochemical analyses revealed that the positive area of MPO, which is a neutrophil marker, was markedly decreased in APAP-treated Mincle KO mice (Fig. 4B). Furthermore, compared with WT mice, decreased expression of neutrophilattractant chemokines such as CXC motif chemokine ligand (CXCL) 1 and CXCL2 were observed in the livers of Mincle KO mice (Fig. 4C). These data indicated that Mincle regulated intrahepatic neutrophil infiltration in APAP-treated mice.

Inhibition of Mincle Downstream Signaling Attenuates APAP-Induced Liver Injury. To further determine the role of the Mincle signaling pathway in APAP hepatotoxicity, WT mice were orally treated with GS9973, an inhibitor of Syk, before the administration of APAP. GS9973-treated mice showed lower hepatocellular necrosis (Fig. 5A), decreased TUNEL-positive areas (Fig. 5B), and diminished serum levels of ALT and AST (Fig. 5C) compared with vehicle-treated mice. Consistently, inhibition of Syk signaling downregulated hepatic levels of inflammatory cytokines, such as TNF- $\alpha$ and IL-1 $\beta$ (Fig. 5D). Furthermore, GS9973-pretreated mice showed decreased infiltration of neutrophil and decreased positive area of MPO in the liver compared with mice without GS9973 treatment (Fig. 5, E-H). Taken together, these findings revealed that the Mincle-Syk signaling pathway was crucial in the pathogenesis of hepatic injury induced by APAP.

Mincle Signaling in KCs Contributes to the Liver Injury Induced by APAP. As parenchymal cells of liver, hepatocytes occupy most of the total liver volume and execute the majority of the functions of the liver. In the present study, we found that primary hepatocytes from the WT and Mincle KO mice released a similar level of LDH with APAP treatment (Supplemental Fig. 4). Subsequently, several cell populations from the liver of WT mice were isolated to determine the cellular source of Mincle. As shown in Fig. 6A, KCs expressed 
A

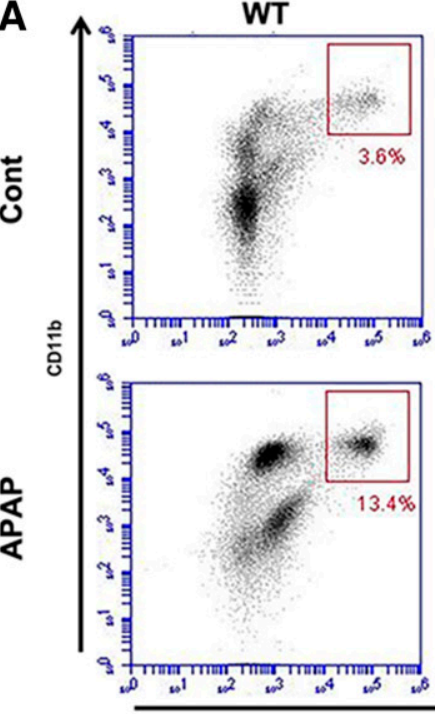

MKO
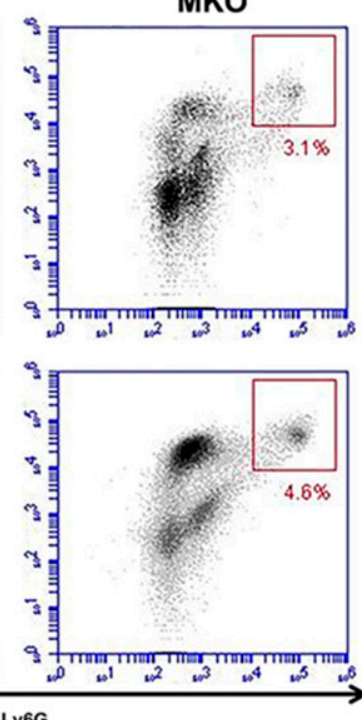

Ly6G

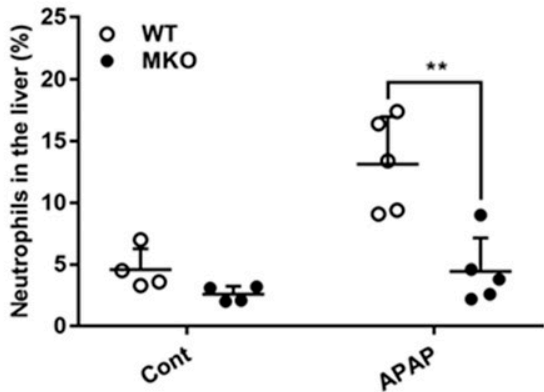

C

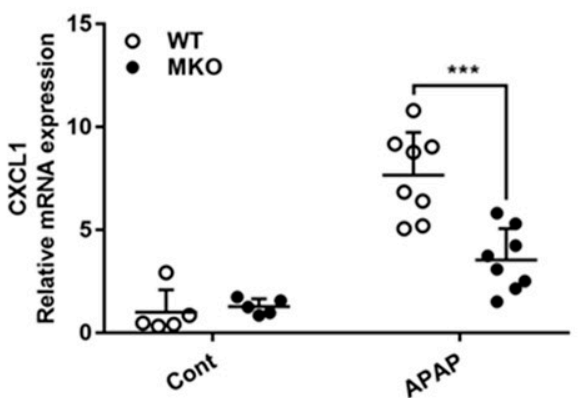

B
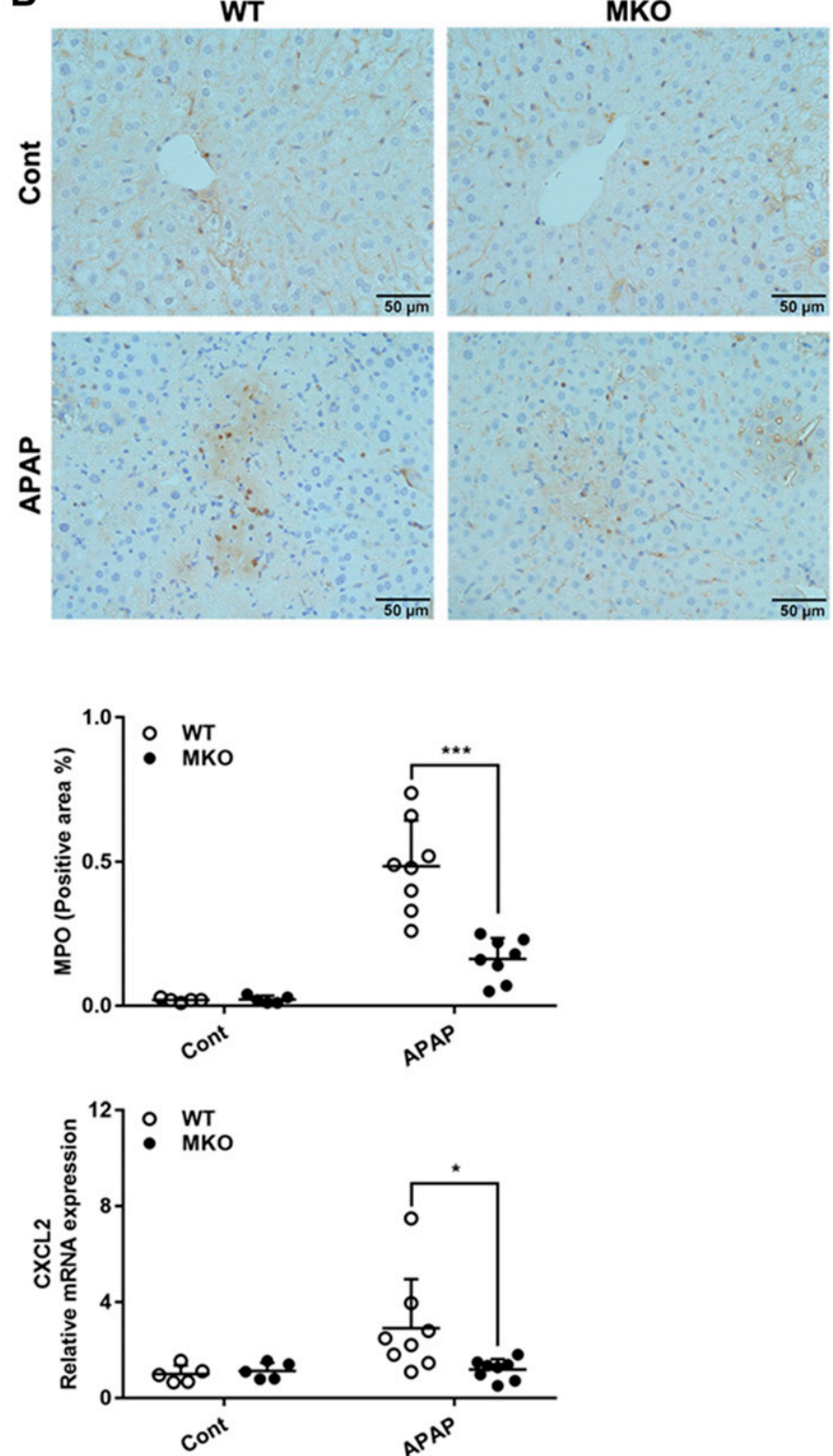

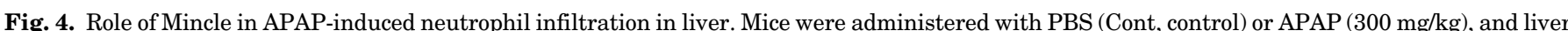

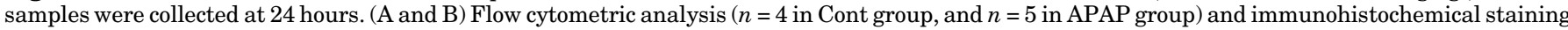

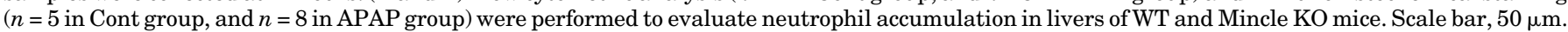

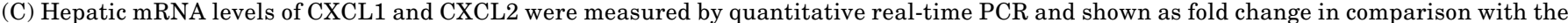

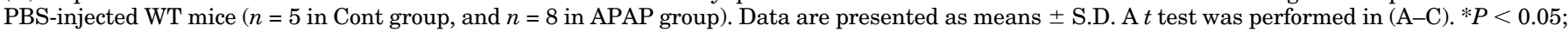
$*_{*} P<0.01 ; * * * P<0.001$ vs. the indicated groups. MKO, Mincle KO.

the highest level of Mincle, signifying that KCs were the major cellular sources of Mincle in the liver. Moreover, after the APAP challenge, the Mincle expression was dramatically upregulated in $\mathrm{KCs}$ (Fig. 6B). Besides, $\mathrm{GdCl}_{3}$-induced $\mathrm{KCs}$ depleted Mincle KO mice showed similar histopathological lesions compared with KC-depleted WT mice (Fig. 6C). The depletion of KCs was confirmed by flow cytometric analysis and qPCR (Supplemental Fig. 5). Consistently, similar TUNEL-positive areas and serum levels of aminotransferases were observed in KC-depleted mice after treatment with $\operatorname{APAP}$ (Fig. 6, D and E), indicating that $\mathrm{KC}$ depletion abolished the detrimental role of Mincle in APAP hepatotoxicity. The $\mathrm{Ct}$ value of GAPDH was represented (Supplemental Fig. 6).
To further investigate the effect of Mincle in KCs on the liver injury induced by APAP, we performed adoptive transfer experiments with F4/80 ${ }^{+} \mathrm{KCs}$ (Fig. 7A). The data showed that the adoptive transfer of $\mathrm{KCs}$ from WT donors partially reversed the hyporesponsiveness to APAP hepatotoxicity in Mincle KO mice, resulting in no statistically significant difference in histopathological lesions, aminotransferase levels, and the TUNEL-positive area (Fig. 7, B-D). Taken together, these results revealed that Mincle signaling was necessitated for the contribution of KCs in APAP-mediated liver injury.

Mincle Signaling Promotes the Inflammatory Response in KCs. Previous studies showed that activated 
A

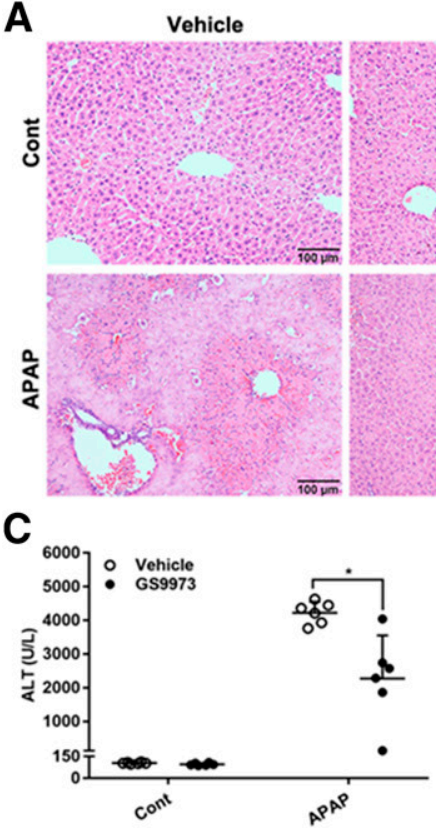

E

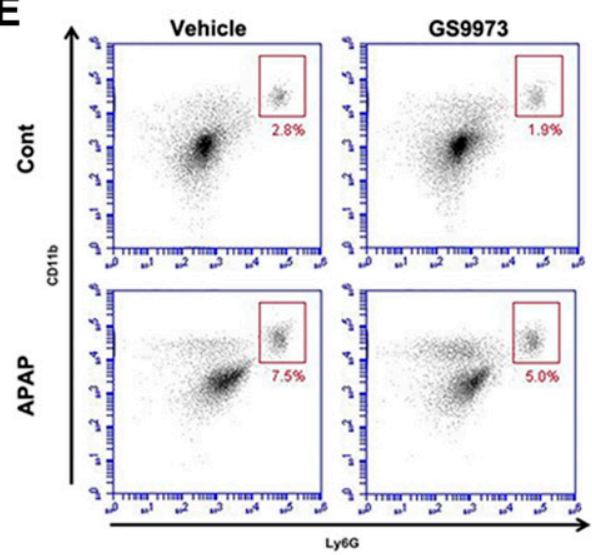

B
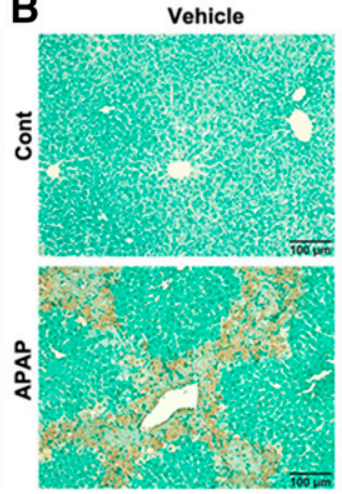

D
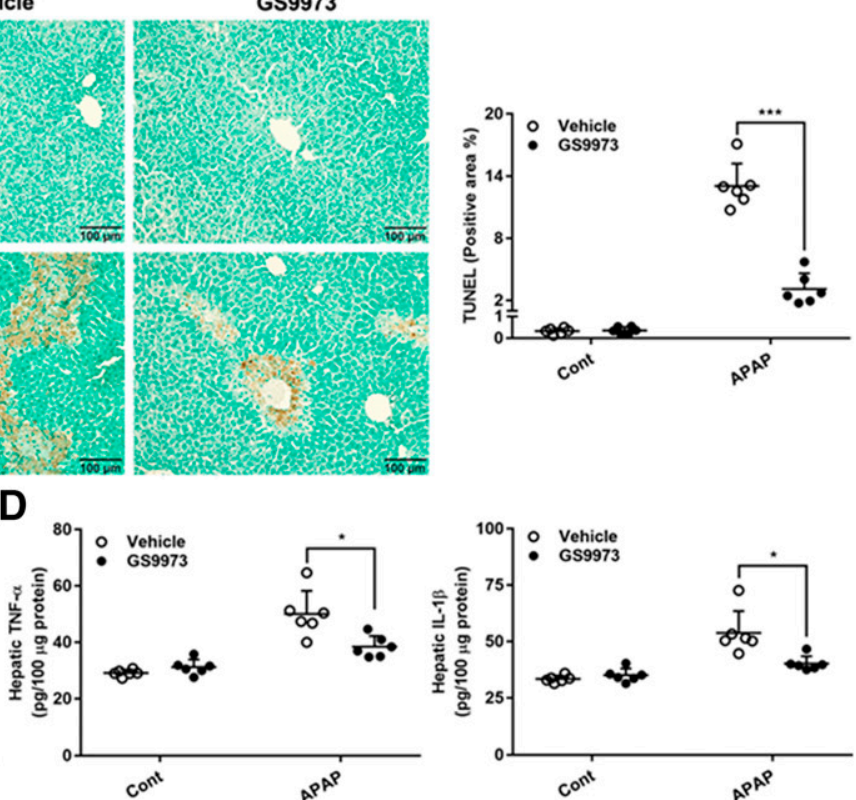

F
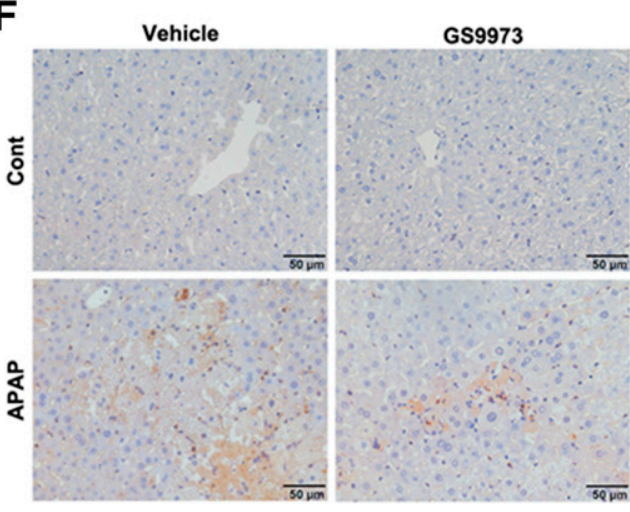

G

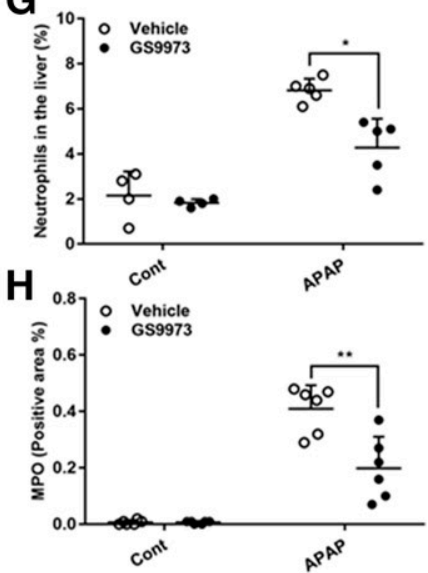

Fig. 5. The effect of Mincle downstream signaling blockade in APAP-induced liver injury. WT mice were injected with PBS (Cont, control) $(n=6)$ or APAP $(300 \mathrm{mg} / \mathrm{kg})(n=6)$ with or without the Syk inhibitor GS9973. Samples were collected at 24 hours after APAP injection. (A) Representative histologic liver sections stained with H\&E are shown. Scale bar, $100 \mu \mathrm{m}$. (B) Apoptosis was determined by TUNEL staining and quantification of TUNELpositive area. Scale bar, $100 \mu \mathrm{m}$. (C) Serum levels of ALT and AST were measured for each cohort. (D) Intrahepatic cytokine levels of TNF- $\alpha$ and IL-1 $\beta$ were measured by ELISA. (E-H) Flow cytometric analysis and immunohistochemical staining were performed to evaluate neutrophil accumulation in mouse livers. Scale bar, $50 \mu \mathrm{m}$. Data are presented as means \pm S.D. A $t$ test was performed in $(\mathrm{B}-\mathrm{D}, \mathrm{G}$, and $\mathrm{H}) . * P<0.05 ; * * P<0.01 ; * * * P<0.001$ vs. the indicated groups.

KCs release a wide array of proinflammatory regulators during APAP hepatotoxicity to modulate liver injury (Jaeschke et al., 2012; Krenkel et al., 2014). We found that when compared with KCs from APAP-injected WT mice, those from APAP-injected Mincle KO mice had decreased mRNA expression levels of IL- $1 \beta$, CXCL1, and CXCL2 (Fig. 8A), which are involved in the inflammatory response and neutrophil infiltration. Moreover, primary KCs treated with a Mincle ligand (TDB) had increased mRNA expression of IL-1 $\beta$, CXCL1, and CXCL2 in WT groups compared with Mincle KO groups (Fig. 8B).

SAP130 released by injured hepatocytes is considered as the only nonpathogenic Mincle ligand with well characterized characteristics (Greco et al., 2016). To further confirm whether Mincle ligand produced by injured hepatocytes affects the activation of $\mathrm{KCs}$, we performed the experiments exploring the effects when KCs were exposed to conditioned media obtained from hepatocytes treated with APAP. As shown in Fig. 8C, the hepatocyte cultured media were prepared as control-conditioned media or APAP-conditioned media after 24 hours of incubation. The extremely increased cell damage was observed at 12 and 24 hours in hepatocytes cultured with APAP (Supplemental Fig. 7), and the level of released SAP130 in APAP-conditioned media was increased when compared with those in control-conditioned media (Supplemental Fig. 8). The conditioned media were mixed with fresh media and used to stimulate primary KCs (Fig. 8C). Results showed that WT KCs cultured in APAPconditioned media exhibited substantially higher expressions of IL-1 $\beta$, CXCL1, and CXCL2 when compared with Mincle KO KCs (Fig. 8D). The Ct value of GAPDH was represented (Supplemental Fig. 9). Collectively, these data 
A

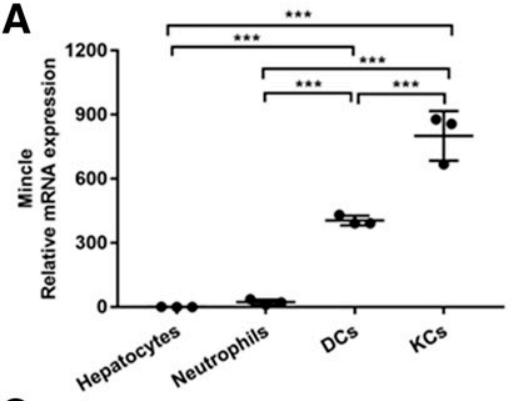

C
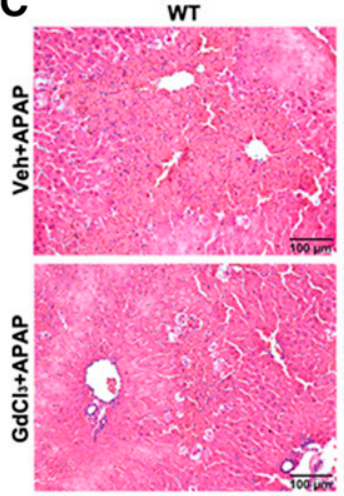

E

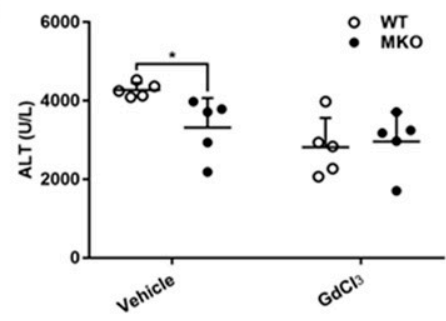

B

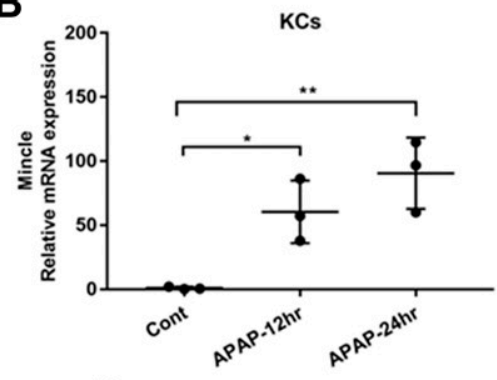

D
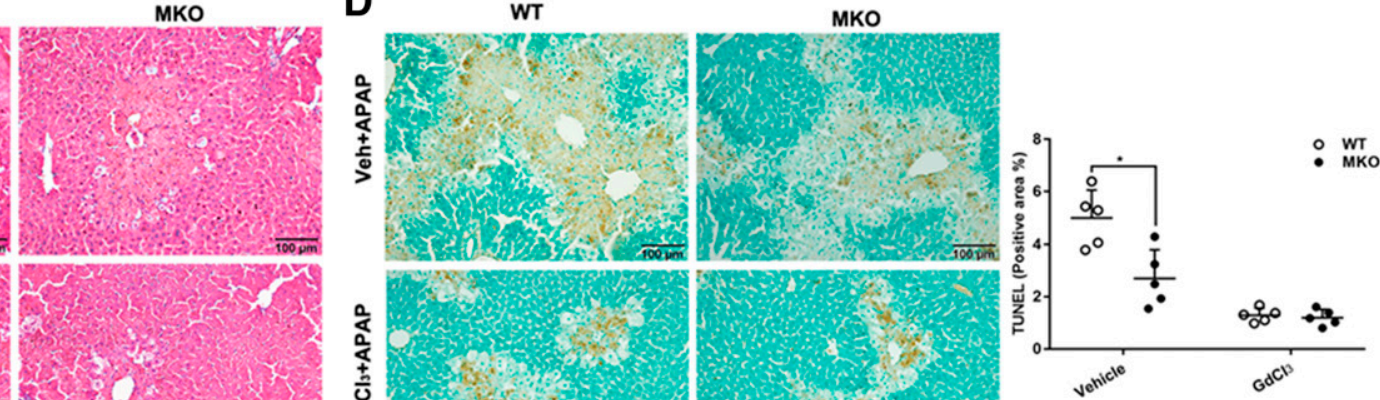

: ${ }_{\text {мко }}^{\text {wT }}$

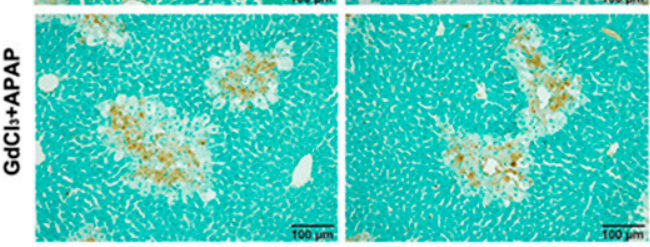

WT
MKO

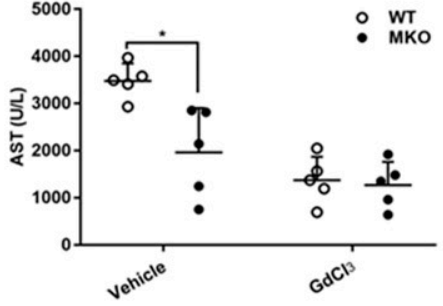

Fig. 6. Role of KCs in the detrimental effect of Mincle in APAP-induced liver injury. (A) Relative mRNA levels of Mincle were measured in isolated liver cell fractions, including hepatocytes, neutrophils, KCs, and DCs in WT mice. Data are shown as fold change compared with the relative expression of Mincle in hepatocytes. (B) Mice were treated with PBS (Cont, control) or APAP (300 mg/kg). KCs were isolated at the indicated times, and relative mRNA levels of Mincle were measured. Data are shown as fold change compared with the relative expression of Mincle in control KCs. (C-E) Mice were intravenously injected with saline as vehicle $(n=5)$ or $\mathrm{GdCl}_{3}(n=5)$ to deplete KCs. Subsequently, APAP $(300 \mathrm{mg} / \mathrm{kg})$ was injected into mice for $24 \mathrm{hours}$. (C) Representative histologic liver sections stained with H\&E are shown. (D) Apoptosis was determined by TUNEL staining and quantification of TUNEL-positive area. (E) Serum levels of ALT and AST were measured. Data are presented as means \pm S.D. A one-way ANOVA with Tukey's post hoc test was performed in (A and B), and $t$ test was performed in (D and $\mathrm{E}) . * P<0.05$; $* * P<0.01 ; * * P<0.001$ vs. the indicated groups. Scale bar, $100 \mu \mathrm{m}$. MKO, Mincle KO.

suggested that stimulating Mincle signaling promoted the inflammatory response in KCs.

\section{Discussion}

The evidence from this study suggests that Mincle deletion and subsequent downstream molecular inhibition attenuates the liver injury induced by APAP. In particular, Mincle in KCs plays a vital role in the progression of APAP hepatotoxicity. Consequently, the findings provide a valuable therapeutic target in acute liver injury and provide insights into the relevance of Mincle in APAP hepatotoxicity.

APAP is one of the most common causes of acute liver injury, and the injury phases can be divided into two steps (Bhushan and Apte, 2019). The first step is the initiation of APAP hepatotoxicity, which involves CYP2E1-mediated catalysis of APAP to a highly toxic metabolite, NAPQI, and the toxicity of NAPQI can be relieved by binding to GSH. The second step is sterile inflammation, which is triggered by DAMPs released by necrotic hepatocytes. It is well described that before APAP administration in a mouse model, a fasting period between 12 and 16 hours in duration could deplete hepatocytic GSH and create comparable conditions for APAP metabolization (Mossanen and Tacke, 2015). A prolonged fasting period could enhance the susceptibility of mouse liver to APAP through decreasing hepatic GSH concentrations and detoxification capacity of the liver (Strubelt et al., 1981). Although Kataoka et al. (2014) have shown that Mincle-deficient mice developed a similar ALT level as WT mice after treatment with APAP, the differences in fasting duration compared with our study may contribute to the resultant discrepancy in results. In the latter study, the mice were prohibited from eating food for 18 hours before and 4 hours after APAP injection. The prolonged fasting time may lead to an aggravation of APAP hepatotoxicity, which could induce some compensatory change in Mincle KO mice (Kataoka et al., 2014) or trigger the inflammation by other signaling such as toll-like receptor signaling (Salama et al., 2015). These results raise a possible hypothesis that the role of Mincle is correlated 
A
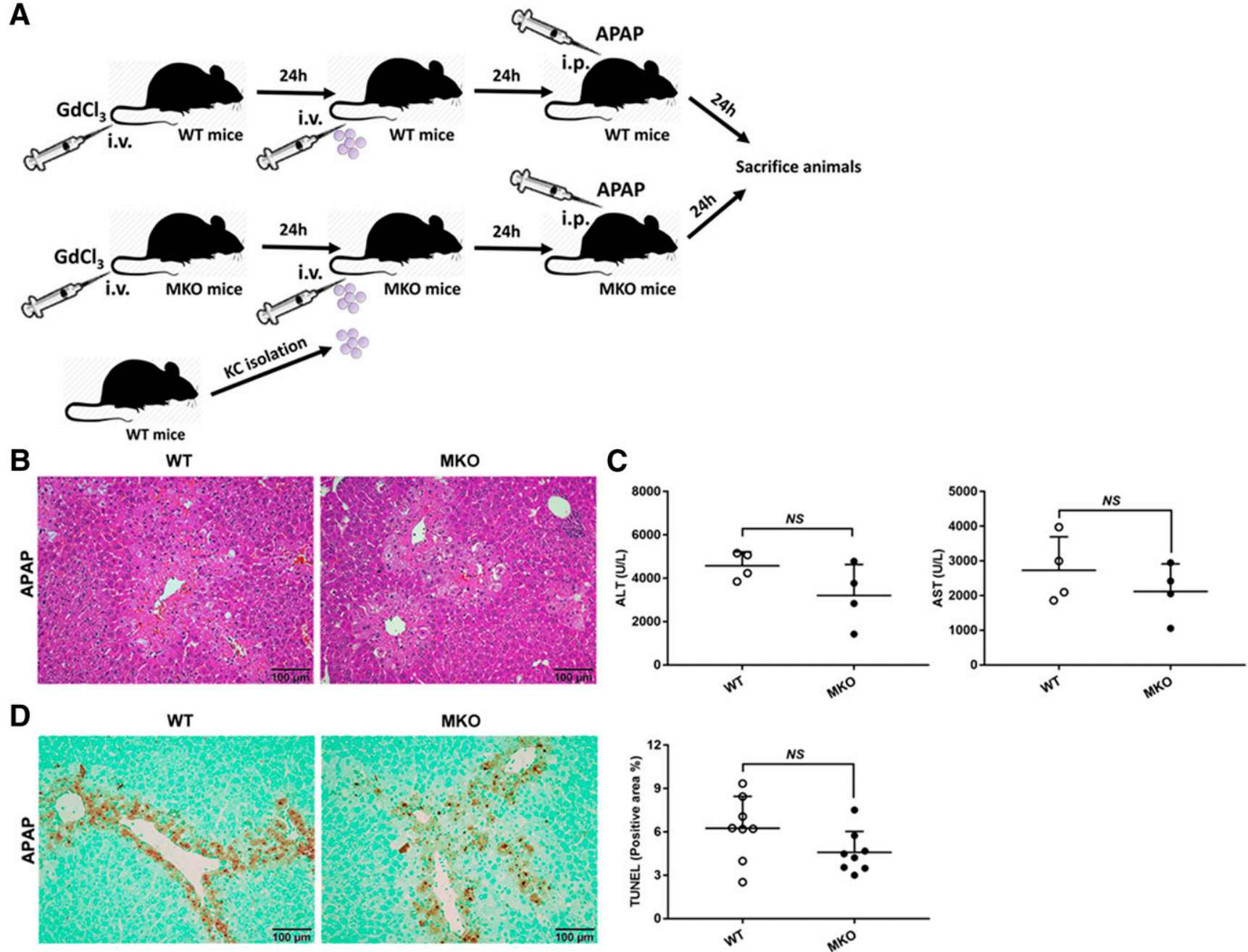

Fig. 7. Role of Mincle signaling in KCs in APAP-induced liver injury. (A) WT mice $(n=4)$ and Mincle KO mice $(n=4)$ were depleted of KCs and adoptively transferred with F4/80 $\mathrm{KCs}\left(1 \times 10^{6}\right.$ cells, intravenous) harvested from WT mice. Mice were then injected with APAP (300 mg/kg) and sacrificed at 24 hours. (B) Representative histologic liver sections stained with H\&E are shown. (C) Serum levels of ALT and AST were measured. (D) Apoptosis was determined by TUNEL staining and quantification of TUNEL-positive area. Data are presented as means \pm S.D. A $t$ test was performed in (C and D). Scale bar, $100 \mu \mathrm{m}$. MKO, Mincle KO; NS, no significant difference.

with the degree of APAP initial hepatotoxicity, and this hypothesis needs to be verified by further research.

In this study, similar levels of GSH and CYP2E1 in the liver were observed in WT and Mincle KO mice, suggesting that Mincle does not substantially modulate the metabolism of APAP. This may be explained by the high expression of Mincle in immune cells but conspicuous absence in hepatocytes (Greco et al., 2016). Of note, we found that KCs expressed the highest level of Mincle in the liver, and APAP-induced injured livers showed increased expression of Mincle in KCs. The main source of proinflammatory cytokines in the liver is believed to be KCs known as residential macrophages (Ju et al., 2002). Our results showed similar severity of APAP hepatotoxicity between KC-depleting WT mice and KCdepleting Mincle $\mathrm{KO}$ mice, indicating that $\mathrm{KCs}$ are required for the detrimental role of Mincle in APAP hepatotoxicity. Connolly et al. (2011) have shown that DC protects against APAP-mediated liver injury. Our findings showed that DCs had a high expression of Mincle. The Mincle expressed in DCs is demonstrated to be activated by stimuli from fungi, mycobacteria, or even parasites and is well related to T-cell differentiation and activation (Iborra et al., 2016; MartinezLopez et al., 2019; Negi et al., 2019). A recent study has reported that the Mincle-Syk axis in DCs could sense mucosalassociated bacteria and induce the release of cytokines such as IL-6, resulting in enhanced intestinal immune barrier function (Martinez-Lopez et al., 2019). Therefore, we believe that Mincle may exert a distinct role depending on specific cell types and different disease models.

In the present study, Mincle was demonstrated as an important modulator of $\mathrm{KC}$ activation. This notion was advocated by a previous study showing that SAP130 was able to extremely increase the production of proinflammatory mediators in WT macrophages primed by low-dose lipopolysaccharide (Zhou et al., 2016). Although the accurate role of $\mathrm{KCs}$ in the progression of acute liver injury remains controversial according to the literature (Ju et al., 2002), it is well accepted that KCs activated by DAMPs could trigger and inappropriately amplify inflammation (Yang and Tonnesseen, 2019). A great deal of evidence has shown that the activation of 
A
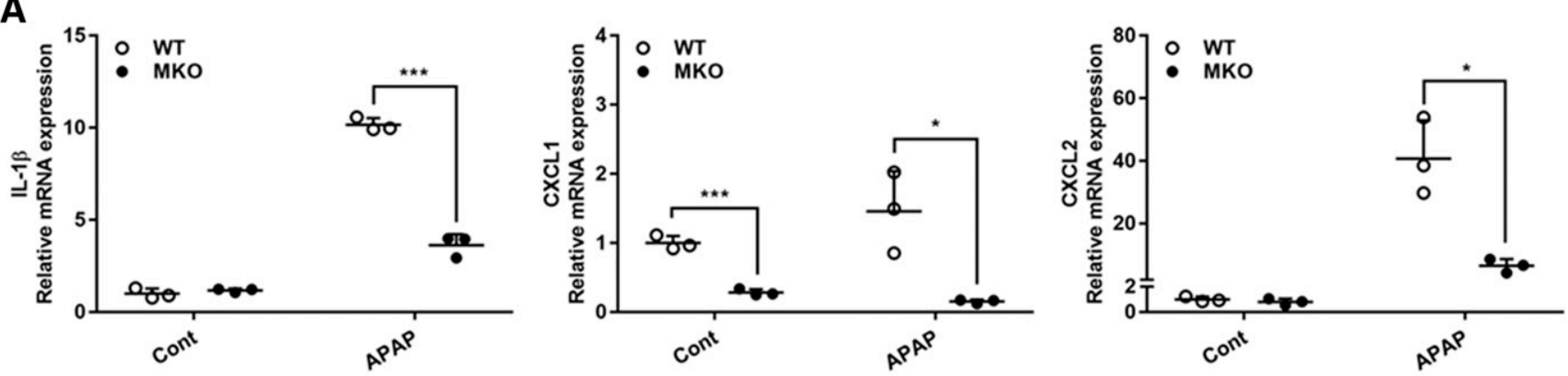

B
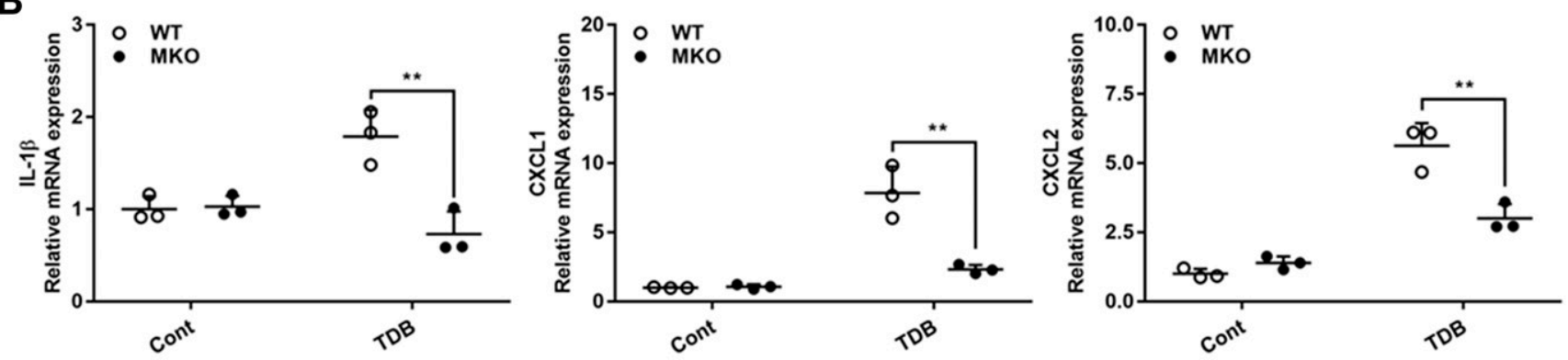

C

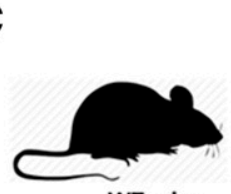

Hepatocyte isolation

WT mice

Cultured in Control or APAP media for $24 \mathrm{~h}$
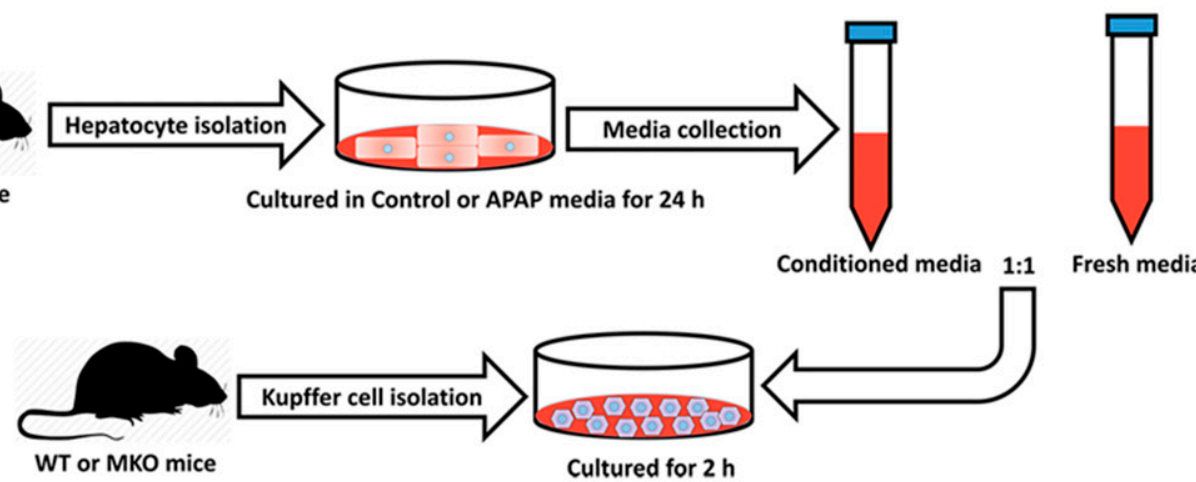

Fresh media
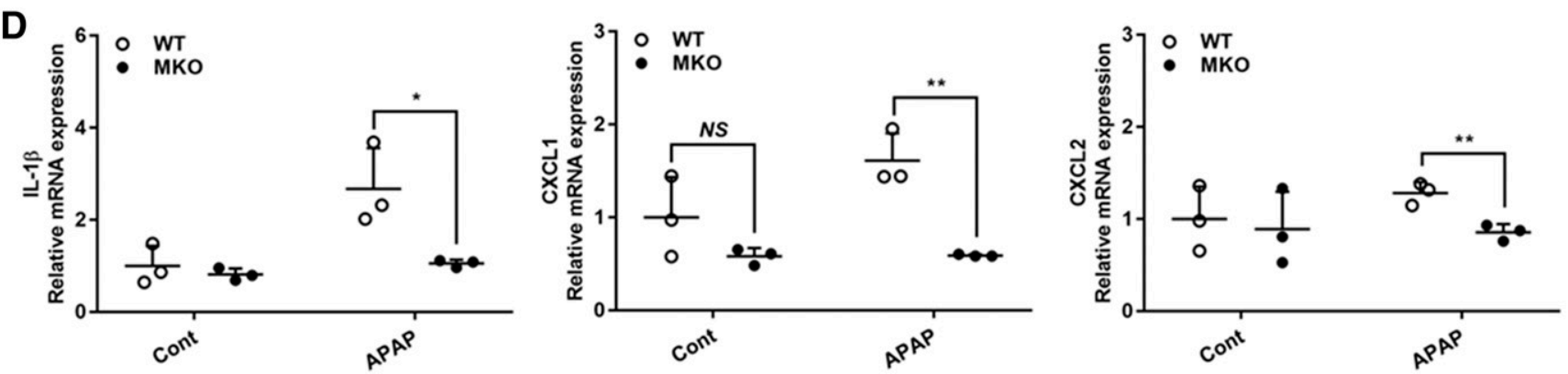

Fig. 8. Role of Mincle signaling in the activation of KCs. (A) KCs were isolated from livers of the two types of mice after PBS (Cont, control) or APAP $(300 \mathrm{mg} / \mathrm{kg})$ injection for 24 hours. Relative mRNA levels of IL-1 $\beta$, CXCL1, and CXCL2 were measured by real-time quantitative PCR and shown as fold change in comparison with the PBS-injected WT mice. (B) Primary KCs isolated from WT and Mincle KO (MKO) mice were treated with Mincle ligand (TDB, $4 \mu \mathrm{g} / \mathrm{ml}$ ) for 24 hours. Relative mRNA levels of IL-1 $\beta$, CXCL1, and CXCL2 were measured by quantitative real-time PCR and shown as fold change in comparison with control WT KCs. (C) Hepatocytes were isolated from WT mice and cultured in media with or without APAP (10 mM). The supernatant was collected as conditioned media at 24 hours. Diluted conditioned media with fresh media was used to stimulate the KCs from WT or Mincle KO mice for 2 hours. (D) Relative mRNA levels of IL-1 $\beta$, CXCL1, and CXCL2 in KCs were measured by quantitative real-time PCR and shown as fold change in comparison with WT KCs treated with control-conditioned media. Data are presented as means \pm S.D. ( $n=3$ independent experiments). A $t$ test was performed in $(\mathrm{A}, \mathrm{B}$, and $\mathrm{D}) . * P<0.05 ; * * P<0.01 ; * * * P<0.001$ vs. the indicated groups. $N S$, no significant difference.

the nucleotide-binding domain and leucine-rich repeat protein 3 (NLRP3) inflammasome is required for the Mincle-induced Syk-mediated upregulation of cytokine production (Gross et al., 2009; Schweneker et al., 2013; Zhang et al., 2015). A previous study also showed that NLRP3-deficient KCs treated with SAP130 had decreased expression of IL- $1 \beta$ compared with WT KCs (Kim et al., 2018). Furthermore, de Castro-Jorge et al. (2019) have reported that the levels of cytokines and the population of infiltrated neutrophils were decreased in the footpad of Mayaro virus-infected NLRP3-deficient mice. 


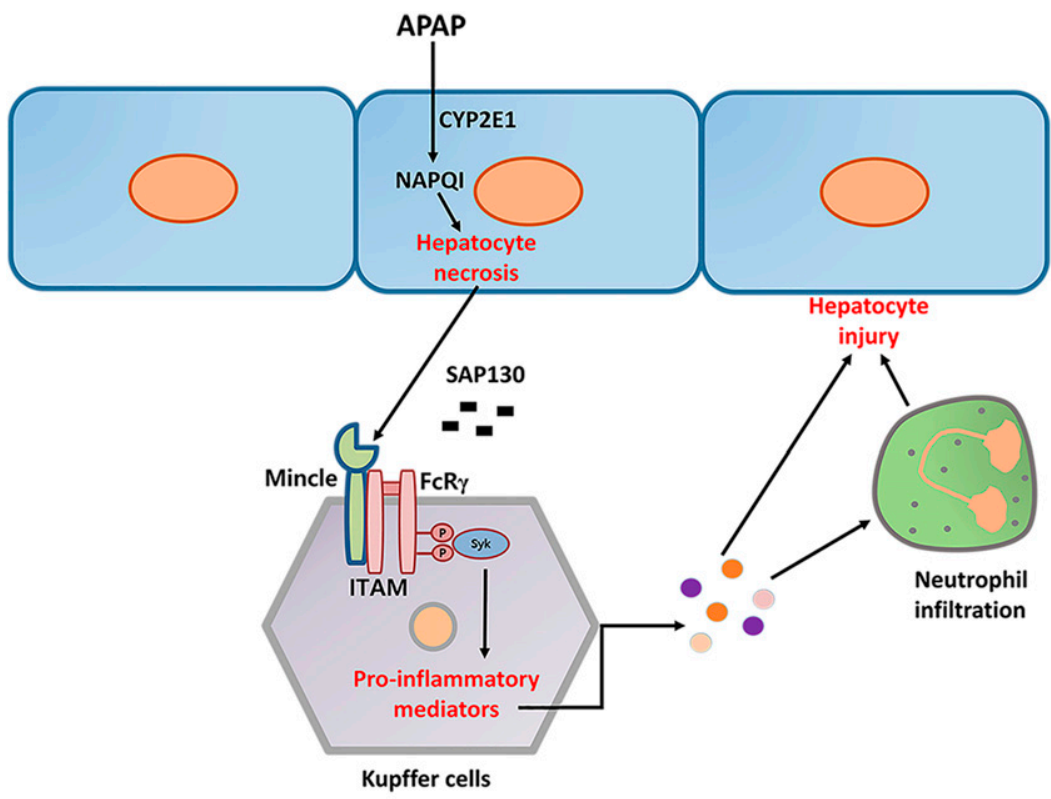

Fig. 9. Schematic presentation showing the detrimental role of Mincle in APAP-induced liver injury. Mincle signaling activates Kupffer cells to produce proinflammatory mediators, which in turn promote hepatocyte damage and neutrophil infiltration. Thus, manipulating the Mincle signaling might be useful as a novel therapeutic strategy to treat APAP-induced liver injury. CYP2E1, cytochrome P450 2E1; FcR $\gamma$, Fc receptor $\gamma$-chain; ITAM, immunoreceptor tyrosine-based activation motif.

Figure 9

Therefore, further study is needed to explore whether an NLRP3-dependent manner is involved in the detrimental role of Mincle in APAP hepatotoxicity.

Collectively, as shown in the schematic (Fig. 9), the present work indicates a regulatory role of Mincle in KC activation, which contributes to the full progression of liver injury induced by APAP. It is hypothesized that understanding this mechanism will offer valuable insights to overcome the limitation of APAP hepatotoxicity treatment.

\section{Authorship Contributions}

Participated in research design: Zhao, J.-W. Kim, Han, B. Kim. Conducted experiments: Zhao, J.-W. Kim, Zhou, Tian.

Performed data analysis: Zhao, Qi, Lim.

Wrote or contributed to the writing of the manuscript: Zhao, Zhou, B. Kim.

\section{References}

Bhushan B and Apte U (2019) Liver regeneration after acetaminophen hepatotoxicity: mechanisms and therapeutic opportunities. Am J Pathol 189:719-729. Brown GD (2008) Sensing necrosis with Mincle. Nat Immunol 9:1099-1100.

Chatterjee S, Ganini D, Tokar EJ, Kumar A, Das S, Corbett J, Kadiiska MB, Waalkes MP, Diehl AM, and Mason RP (2013) Leptin is key to peroxynitrite-mediated oxidative stress and Kupffer cell activation in experimental non-alcoholic steatohepatitis. J Hepatol 58:778-784.

Chen L, Wang P, Manautou JE, and Zhong XB (2020) Knockdown of long noncoding RNAs hepatocyte nuclear factor $1 \alpha$ antisense RNA 1 and hepatocyte nuclear factor $4 \alpha$ antisense RNA 1 alters susceptibility of acetaminophen-induced cytotoxicity in HepaRG cells. Mol Pharmacol 97:278-286.

Chun LJ, Tong MJ, Busuttil RW, and Hiatt JR (2009) Acetaminophen hepatotoxicity and acute liver failure. J Clin Gastroenterol 43:342-349.

Connolly MK, Ayo D, Malhotra A, Hackman M, Bedrosian AS, Ibrahim J, CiezaRubio NE, Nguyen AH, Henning JR, Dorvil-Castro M, et al. (2011) Dendritic cell depletion exacerbates acetaminophen hepatotoxicity. Hepatology 54:959-968.

Currie KS, Kropf JE, Lee T, Blomgren P, Xu J, Zhao Z, Gallion S, Whitney JA, Maclin

D, Lansdon EB, et al. (2014) Discovery of GS-9973, a selective and orally efficacious inhibitor of spleen tyrosine kinase. $J$ Med Chem 57:3856-3873.

de Castro-Jorge LA, de Carvalho RVH, Klein TM, Hiroki CH, Lopes AH, Guimarães RM, Fumagalli MJ, Floriano VG, Agostinho MR, Slhessarenko RD, et al. (2019) The NLRP3 inflammasome is involved with the pathogenesis of Mayaro virus. PLoS Pathog 15:e1007934.

de Rivero Vaccari JC, Brand FJ III, Berti AF, Alonso OF, Bullock MR, and de Rivero Vaccari JP (2015) Mincle signaling in the innate immune response after traumatic brain injury. $J$ Neurotrauma 32:228-236

Greco SH, Torres-Hernandez A, Kalabin A, Whiteman C, Rokosh R, Ravirala S, Ochi A, Gutierrez J, Salyana MA, Mani VR, et al. (2016) Mincle signaling promotes Con A hepatitis. J Immunol 197:2816-2827.
Gross O, Poeck H, Bscheider M, Dostert C, Hannesschläger N, Endres S, Hartmann G, Tardivel A, Schweighoffer E, Tybulewicz V, et al. (2009) Syk kinase signalling couples to the Nlrp3 inflammasome for anti-fungal host defence. Nature 459:433-436.

He Y, Xu L, Li B, Guo ZN, Hu Q, Guo Z, Tang J, Chen Y, Zhang Y, Tang J, et al. (2015) Macrophage-inducible C-type lectin/spleen tyrosine kinase signaling pathway contributes to neuroinflammation after subarachnoid hemorrhage in rats. Stroke 46:2277-2286.

Holt MP, Cheng L, and Ju C (2008) Identification and characterization of infiltrating macrophages in acetaminophen-induced liver injury. $J$ Leukoc Biol 84: 1410-1421.

Iborra S, Martínez-López M, Cueto FJ, Conde-Garrosa R, Del Fresno C, Izquierdo HM, Abram CL, Mori D, Campos-Martín Y, Reguera RM, et al. (2016) Leishmania uses Mincle to target an inhibitory ITAM signaling pathway in dendritic cells that dampens adaptive immunity to infection. Immunity 45:788-801.

Ishikawa E, Ishikawa T, Morita YS, Toyonaga K, Yamada H, Takeuchi O, Kinoshita T, Akira S, Yoshikai Y, and Yamasaki S (2009) Direct recognition of the mycobacterial glycolipid, trehalose dimycolate, by C-type lectin Mincle. J Exp Med 206: 2879-2888.

Jaeschke H and Bajt ML (2006) Intracellular signaling mechanisms of acetaminopheninduced liver cell death. Toxicol Sci 89:31-41.

Jaeschke H, Ramachandran A, Chao X, and Ding WX (2019) Emerging and established modes of cell death during acetaminophen-induced liver injury. Arch Toxicol 93:3491-3502.

Jaeschke H, Williams CD, Ramachandran A, and Bajt ML (2012) Acetaminophen hepatotoxicity and repair: the role of sterile inflammation and innate immunity. Liver Int 32:8-20.

Ju C, Reilly TP, Bourdi M, Radonovich MF, Brady JN, George JW, and Pohl LR (2002) Protective role of Kupffer cells in acetaminophen-induced hepatic injury in mice. Chem Res Toxicol 15:1504-1513.

Kataoka H, Kono H, Patel Z, Kimura Y, and Rock KL (2014) Evaluation of the contribution of multiple DAMPs and DAMP receptors in cell death-induced sterile inflammatory responses [published correction appears in PLoS One (2014) 9: e113029]. PLoS One 9:e104741.

Kim JW, Roh YS, Jeong H, Yi HK, Lee MH, Lim CW, and Kim B (2018) Spliceosomeassociated protein 130 exacerbates alcohol-induced liver injury by inducing NLRP3 inflammasome-mediated IL-1 $\beta$ in mice. Am $J$ Pathol 188:967-980.

Krenkel O, Mossanen JC, and Tacke F (2014) Immune mechanisms in acetaminophen-induced acute liver failure. Hepatobiliary Surg Nutr 3:331-343.

Lancaster EM, Hiatt JR, and Zarrinpar A (2015) Acetaminophen hepatotoxicity: an updated review. Arch Toxicol 89:193-199.

Larsen FS and Wendon J (2014) Understanding paracetamol-induced liver failure. Intensive Care Med 40:888-890.

Larson AM, Polson J, Fontana RJ, Davern TJ, Lalani E, Hynan LS, Reisch JS, Schiødt FV, Ostapowicz G, Shakil AO, et al.; Acute Liver Failure Study Group (2005) Acetaminophen-induced acute liver failure: results of a United States multicenter, prospective study. Hepatology 42:1364-1372.

Lawson JA, Farhood A, Hopper RD, Bajt ML, and Jaeschke H (2000) The hepatic inflammatory response after acetaminophen overdose: role of neutrophils. Toxicol Sci 54:509-516.

Lee WB, Kang JS, Yan JJ, Lee MS, Jeon BY, Cho SN, and Kim YJ (2012) Neutrophils promote mycobacterial trehalose dimycolate-induced lung inflammation via the Mincle pathway. PLoS Pathog 8:e1002614.

Lin J, He K, Zhao G, Li C, Hu L, Zhu G, Niu Y, and Hao G (2017) Mincle inhibits neutrophils and macrophages apoptosis in A. fumigatus keratitis. Int Immunopharmacol 52:101-109. 
Martin-Murphy BV, Holt MP, and Ju C (2010) The role of damage associated molecular pattern molecules in acetaminophen-induced liver injury in mice. Toxicol Lett 192:387-394.

Martinez-Lopez M, Iborra S, Conde-Garrosa R, Mastrangelo A, Danne C, Mann ER, Reid DM, Gaboriau-Routhiau V, Chaparro M, Lorenzo MP, et al. (2019) Microbiota sensing by Mincle-Syk axis in dendritic cells regulates interleukin-17 and -22 production and promotes intestinal barrier integrity. Immunity 50:446-461.e9.

Matsumoto M, Tanaka T, Kaisho T, Sanjo H, Copeland NG, Gilbert DJ, Jenkins NA, and Akira S (1999) A novel LPS-inducible C-type lectin is a transcriptional target of NF-IL6 in macrophages. J Immunol 163:5039-5048.

Merlin S, Bhargava KK, Ranaldo G, Zanolini D, Palestro CJ, Santambrogio L, Prat M, Follenzi A, and Gupta S (2016) Kupffer cell transplantation in mice for elucidating monocyte/macrophage biology and for potential in cell or gene therapy. Am $J$ Pathol 186:539-551.

Michel MC, Murphy TJ, and Motulsky HJ (2020) New author guidelines for displaying data and reporting data analysis and statistical methods in experimental biology. Mol Pharmacol 97:49-60.

Mossanen JC and Tacke F (2015) Acetaminophen-induced acute liver injury in mice. Lab Anim 49(1 Suppl):30-36.

Negi S, Pahari S, Bashir H, and Agrewala JN (2019) Gut microbiota regulates Mincle mediated activation of lung dendritic cells to protect against Mycobacterium tuberculosis. Front Immunol 10:1142.

Prescott LF, Park J, Ballantyne A, Adriaenssens P, and Proudfoot AT (1977) Treatment of paracetamol (acetaminophen) poisoning with $\mathrm{N}$-acetylcysteine. Lancet 2:432-434.

Richardson MB and Williams SJ (2014) MCL and Mincle: C-type lectin receptors that sense damaged self and pathogen-associated molecular patterns. Front Immunol 5 288.

Salama M, Elgamal M, Abdelaziz A, Ellithy M, Magdy D, Ali L, Fekry E, Mohsen Z, Mostafa M, Elgamal H, et al. (2015) Toll-like receptor 4 blocker as potential therapy for acetaminophen-induced organ failure in mice. Exp Ther Med 10: 241-246.

Schweneker K, Gorka O, Schweneker M, Poeck H, Tschopp J, Peschel C, Ruland J, and Gross O (2013) The mycobacterial cord factor adjuvant analogue trehalose6,6'-dibehenate (TDB) activates the Nlrp3 inflammasome. Immunobiology 218 : $664-673$.
Smilkstein MJ, Knapp GL, Kulig KW, and Rumack BH (1988) Efficacy of oral $\mathrm{N}$-acetylcysteine in the treatment of acetaminophen overdose. Analysis of the national multicenter study (1976 to 1985). N Engl J Med 319:1557-1562.

Strubelt O, Dost-Kempf E, Siegers CP, Younes M, Völpel M, Preuss U, and Dreckmann JG (1981) The influence of fasting on the susceptibility of mice to hepatotoxic injury. Toxicol Appl Pharmacol 60:66-77.

Wu J, Li J, Salcedo R, Mivechi NF, Trinchieri G, and Horuzsko A (2012) The proinflammatory myeloid cell receptor TREM-1 controls Kupffer cell activation and development of hepatocellular carcinoma. Cancer Res 72:3977-3986.

Yamasaki S, Ishikawa E, Sakuma M, Hara H, Ogata K, and Saito T (2008) Mincle is an ITAM-coupled activating receptor that senses damaged cells. Nat Immunol $\mathbf{9}$ : $1179-1188$

Yang R and Tonnesseen TI (2019) DAMPs and sterile inflammation in drug hepatotoxicity. Hepatol Int 13:42-50.

Zaher H, Buters JT, Ward JM, Bruno MK, Lucas AM, Stern ST, Cohen SD, and Gonzalez FJ (1998) Protection against acetaminophen toxicity in CYP1A2 and CYP2E1 double-null mice. Toxicol Appl Pharmacol 152:193-199.

Zhang Z, He L, Hu S, Wang Y, Lai Q, Yang P, Yu Q, Zhang S, Xiong F, Simsekyilmaz $\mathrm{S}$, et al. (2015) AAL exacerbates pro-inflammatory response in macrophages by regulating Mincle/Syk/Card9 signaling along with the Nlrp3 inflammasome assembly. Am J Transl Res 7:1812-1825.

Zhao J, Park S, Kim JW, Qi J, Zhou Z, Lim CW, and Kim B (2020) Nicotine attenuates concanavalin A-induced liver injury in mice by regulating the $\alpha 7$ nicotinic acetylcholine receptor in Kupffer cells. Int Immunopharmacol 78:106071.

Zhou H, Yu M, Zhao J, Martin BN, Roychowdhury S, McMullen MR, Wang E, Fox PL, Yamasaki S, Nagy LE, et al. (2016) IRAKM-Mincle axis links cell death to inflammation: pathophysiological implications for chronic alcoholic liver disease. Hepatology 64:1978-1993.

Address correspondence to: Dr. Bumseok Kim, College of Veterinary Medicine, Jeonbuk National University, Iksan, Republic of Korea 54531. E-mail: bskims@jbnu.ac.kr; or Dr. Kang Min Han, Department of Pathology, Dongguk University Ilsan Hospital, Goyang, Republic of Korea 10326. E-mail: kiekie53@hanmail.net 